\title{
INFLUENCIA DE LOS RECURSOS DIGITALES EN LA ENSEÑANZA Y EL APRENDIZAJE DEL IDIOMA INGLÉS EN LAS ESCUELAS NORMALES DE OAXACA, MÉXICO
}

\section{INFLUENCE OF DIGITAL RESOURCES IN TEACHING AND LEARNING ENGLISH IN NORMAL SCHOOLS OF OAXACA, MÉXICO}

\section{Karen Josefa González Vega ${ }^{1}$}

Universidad La Salle, Oaxaca, México

\section{RESUMEN}

En el presente artículo se establece una investigacióncuyoobjetivoconsisteen determinar la influencia que tienen los recursos digitales en

1 Maestrante en Educación Superior, en la Universidad La Salle Oaxaca, México. Lic. en Educación Primaria, Lic. en Enseñanza de Lenguas Extrajeras, docente en Educación Primaria. Línea de Investigación: Bilingüísmo en la formación docente. kanegonz20@gmail. com , ORCID: https://orcid.org/0000-0003-0680-5515

Google Académico: https://scholar.google.com.co/citation s?hl=es\&user=i1PXJbkAAAAJ\&view op=list works\&citft $=1 \&$ citft=2\&citft=3\&email for_op=kanegonz20\%40gmail. com\&gmla =AJsN-F5dOn0qkEJdEKL3k7P7Yish3VMU7n7xAo1ROe8bC-B 5IZm8Zcf6r1mQ77HcMw4NOTqzNom4NnxAqDLpfeBf3ba6mTQxhEOfPKKsBqIPCzm60_vIPYij4_eC1odhGSk9Eny2pULNfSa4I3RvoUh2PyGqk6Lov6YCVgOOBcBZzbiUIZSHKN8PdHBrqMJ5V2pAMszh8p8P30RgsV7YcL q0MJXCOrerM9T8W-x7ww 35Aakt8aPI heLjrrrDw6zFYEpoUtm la enseñanza y aprendizaje del idioma Inglés en las Escuelas Normales de Valles Centrales del estado de Oaxaca, México. Se articula en datos referidos de los profesores de lengua extranjera de este nivel educativo, al implementar los programas digitales en la formación de docentes. Así mismo, se incluyen las opiniones de los alumnos que cursan la asignatura, para advertir si éstos consideran que las recursos digitales favorecen sus propios procesos de aprendizaje de este idioma. El enfoque metodológico que se siguió fue la encuesta. Se determina en la existencia de vinculación entre la Educación y los recursos digitales, para 
advertir si éstos consideran que los recursos digitales favorecen sus propios procesos de aprendizaje de este idioma. El enfoque metodológico que se siguió fue la encuesta, se determina en la existencia de vinculación entre la Educación y los recursos digitales, que constituyen una práctica de formación integral del estudiante a través de una metodología de enseñanza innovadora del lenguaje, que permita la reflexión y enriquecimiento al relacionarse con el conocimiento, propendiendo así por una educación de calidad.

PALABRAS CLAVE: Recursos digitales, enseñanza, aprendizaje, lengua extranjera.

\section{ABSTRACT}

In this article an investigation establishes whose objective is to determine the influence of digital resources in teaching and learning English in Normal Schools of Central Valleys of Oaxaca, Mexico, it is divided into data on the foreign language teachers of this level, to implement digital programs in teacher training. It also includes the opinions of students attending the course, to warn if they consider that digital resources favor their own processes of learning this language. The methodological approach followed was the surveys; it is determined by the existence of links between education and digital resources, which constitute a practice of formation of the student, through an innovative methodology of teaching language, allowing reflection and enrichment as they relate to knowledge, achieving quality education.

KEYWORDS: Digital resources, teaching, learning, foreign language.

\section{INTRODUCCIÓN}

Actualmente el planeta Tierra está conformado por una sociedad que se va desarrollando conforme pasa el tiempo. Así como la tecnología va mejorando y cambiando, también las ideologías se van modificando, por lo que se precisa instruir a los jóvenes en la adquisición de conocimientos y estrategias de pensamiento para integrarse mejor a una sociedad cada vez más desarrollada, ya que son el futuro del país y de cada individuo depende el rumbo que se le dé a ese porvenir.

La Universidad Nacional Autónoma de México (UNAM) (2012) plantea que las dos décadas transcurridas desde la incorporación de las escuelas normales a la educación superior han evidenciado tensiones, expresadas de muy diversas formas, que abonan a favor de un sentimiento de desplazamiento ante descalificaciones que enfatizan sus debilidades académicas institucionales, que desalientan en vez de entusiasmar el proceso de cambio.

A pesar de las políticas de la educación normalista y del programa de fortalecimiento de la educación normal, el sistema no ha avanzado en el desarrollo de las funciones establecidas en su propósito como instituciones educativas de nivel superior. Una de las debilidades de este sector es en ámbito de investigación, debido a la insuficiencia de formación del personal académico.

México carece de una estrategia nacional precisa sobre el tema del dominio del idioma inglés como instrumento para generar la competitividad y la movilidad social. Recientemente surge el interés de incorporar una política nacional que favorezca el aprendizaje del idioma inglés, la escasez de maestros especializados en esta disciplina, ha forjado que el impulso sea menor de lo esperado y su cobertura es limitada, a pesar que se imparte del idioma inglés en la educación básica de diversos estados de la República Mexicana.

De acuerdo con el Plan de estudios 2012 que establece la Secretaría de Educación Pública (SEP) en las escuelas de Educación Normal (SEP, 2012), la asignatura de Lengua adicional y Tecnologías de la información y la comunicación se compone de 7 cursos que 
integran actividades de docencia de tipo teóricopráctico, con una carga de 4 horas semanales y un valor de 4.5 créditos académicos cada uno, estableciendo dichas asignaturas a partir del tercer semestre de las Licenciaturas, en donde el propósito es que los alumnos tengan conocimientos básicos de la lengua que les permita comunicarse de manera sencilla tanto en forma oral como escrita en contextos familiares y cercanos a su entorno personal, convirtiendo al futuro maestro en estudiante de una segunda lengua, y desarrollar indirectamente ciertas aptitudes que le pueden ser de gran utilidad para su vida como profesional de la docencia, dejando a un lado las estrategias metodológicas que implican la enseñanza de una lengua extranjera.

Es prioritario reorientar las teorías y metodologías de aprendizaje e incorporación de la tecnología en la enseñanza de una lengua extranjera, evitando las malas prácticas formativas donde los recursos tecnológicos son incorporados por el propio sistema educativo, y más que servir de cambio, innovación y transformación educativa, sirven para la perpetuación de un modelo bancario de educación y formación.

Resulta evidente que el sentido más genuino de educación se ocupa de las acciones de los docentes que tienen como destinatarios directos a los estudiantes, y que esa forma de realizar la educación ha sido fundamental en la generación histórica de los usos prácticos que comprenden la tradición pedagógica, expresando la forma más humana de comunicar a otros las elaboraciones de la cultura. (Sacristán, 1998).

La intención de este estudio no es establecer juicios de valor, ni decir lo que hay que hacer frente al campo problemático abordado, sino de demostrar problemas, presentar evidencias que permitan mirar e genuinamente interpretar la realidad de una manera diferente a lo ya establecido.

\section{MARCO CONTEXTUAL}

\subsection{Las escuelas normales en el estado de Oaxaca.}

Con base en una investigación realizada y publicada por un artículo periodístico (Castillejos, 2014), relata la educación y formación de las escuelas Normales en el estado de Oaxaca en sus inicios, el cual refiere que el 24 de Julio de 1924, se recuerda la reapertura de la Escuela Normal Mixta Federalizada de Oaxaca, convertida en la actualidad en Centro Regional de Educación Normal en Oaxaca.

En la actualidad, existen 11 normales en el estado las cuales ofrecen estudios de Licenciaturas en Educación Preescolar, Primaria, Física, Intercultural Bilingüe; Especial en el área Auditiva y de Lenguaje, así como en el área Intelectual.

Los planteles que ofertan los servicios educativos son la Escuelas Normales de Educación Especial de Oaxaca, de Educación Preescolar de Oaxaca, el Centro Regional de Educación Normal de Oaxaca, Experimental de Huajuapan, Experimental "Presidente Lázaro Cárdenas" y la Experimental de Teposcolula.

Así como la Escuela Normal Experimental" Presidente Venustiano Carranza" en San Juan Cacahuatepec, el Centro Regional de Educación Normal Río Grande, Urbana Federal del Istmo, Bilingüe e Intercultural de Oaxaca y Rural "Vanguardia" en Tlacochahuaya.

\subsection{Plan de estudios 2012, inserción actual en la educación normal.}

La Secretaría de Educación Pública implementa un nuevo Plan de estudios en las escuelas Normales del país, para mejorar la calidad de la formación docente, intelectual y moral de los futuros profesores. En esto radican los nuevos programas que traen inevitablemente a los problemas de fin y contenido, el problema de 
métodos, procedimientos, formas y recursos pedagógicos, que han de aplicarse para realizar eficazmente la formación de los nuevos maestros.

Con la entrada de este nuevo plan de estudios, el papel del docente se modificó y se empezó a dar protagonismo al desarrollo de aprendizajes significativos para los alumnos. La aplicación de dicho plan exige un mecanismo eficaz de seguimiento y evaluación que permita atender con congruencia distintos tipos de problemas, tanto los que se derivan de condiciones inadecuadas para la aplicación del plan, como insuficiencias de la propuesta curricular misma.

En 2011 inició la fase de pilotaje de la Reforma curricular de normales 2012, enfocada a tres tendencias fundamentales:

- El enfoque centrado en el aprendizaje.

- Flexibilidad curricular.

- Enfoque basado en competencias.

El enfoque tradicional de enseñanza-aprendizaje en las escuelas normales, que estaba centrado en la trasmisión de información y en la figura del profesor como fuente casi única del saber tiende a modificarse como resultado, en gran medida, de los diversos estudios sobre las corrientes psicológicas y pedagógicas, para dar paso a nuevos énfasis y orientaciones. Así mismo, la información, sus fuentes y los medios para difundirlo se han diversificado, como consecuencia del avance científico y el desarrollo de las TIC puede entonces hablarse de la emergencia de un nuevo paradigma educativo (SEP, 2011).

La tendencia actual del Plan de estudios 2011, referente al enfoque centrado en el aprendizaje, reconoce la capacidad del sujeto para aprender considerando sus experiencias y conocimientos previos, así como los que se le ofrece por la vía institucional y medios tecnológicos.

\section{MARCO TEÓRICO}

\subsection{Inglés como lengua extranjera en la formación de docentes.}

Hasta el momento, el sistema educativo no está cumpliendo con los requerimientos del sector productivo con respecto a la competencias en lenguas extranjeras. La calidad de enseñanza del idioma inglés, sobre todo en niveles básicos está ligada con la calidad educativa general. Si la calidad educativa de educación básica es deficiente, será difícil que los alumnos tengan un buen desempeño aprendiendo inglés. Es necesario hacer cambios del sistema educativo desde la raíz.

La propuesta establecida por el Instituto Mexicano para la Competitividad (IMCO) (2015) establece el fortalecimiento del currículo de las escuelas normales, la propuesta plantea establecer una restructuración del currículo cuyo objetivo sea que los docentes normalistas egresen de su carrera con el nivel requerido para ejercer como docente de inglés en cualquier nivel educativo.

En un plazo mediano, esta iniciativa permitirá contar con docentes capaces de impartir la asignatura de inglés como lengua extranjera, en su formación deberá incluirse también, como asignatura complementaria, metodología de enseñanza de lenguas extranjeras.

Aunado a esto, es prioritario establecer la posibilidad de exigir como estándar nacional el certificado TKT: Teaching Knowledge Test, desarrollado por Cambridge English Language Assesment, conforme al Marco Común Europeo de Referencia para las Lenguas (MCERL), el cual establece el nivel de expresión, comprensión oral y escrita considerado como Competente 1 (C1) para los egresados de estas escuelas, aunque actualmente se contempla el nivel Independiente 2 (B2). 
Para ello, la materia de inglés debería de ser obligatoria desde el primer semestre de la licenciatura y no desde tercero, como actualmente se plantea en el plan y programa actual. Cada egresado de la escuela normal podría contar con una certificación, misma que deberá mantener vigente a partir de los programas de formación continua que la propia SEP podría proveer.

\subsection{La era digital y su impacto en el sector educativo}

De acuerdo a Podolsky (2014) se establece en su investigación datos del Instituto Nacional de Estadística, Geografía e Informática (INEGI), de los mexicanos que asisten actualmente a la escuela, $56 \%$ utilizan Internet y $69 \%$ usa o ha empleado una computadora en el último año, este estudio se dio con base a una encuesta realizada en el año 2014 estudiantes universitarios del país.

La importancia del manejo de las TIC en el contexto actual es crucial para el desempeño económico del país, si aumenta el acceso a las tecnologías, se espera que crezca la productividad, las horas trabajadas se vuelvan más eficientes, se incrementen los salarios y se fomente el crecimiento económico y la innovación.

Con base en las investigaciones realizadas en este apartado, se determina que hoy en día la enseñanza y aprendizaje del inglés como lengua extranjera es indispensable para el desarrollo humano en diversos sectores sociales, ya que es considerada como una lengua universal, es preocupante que en nuestro país no exista un cambio real en los planes y programas de estudio, siguiendo las mismas prácticas de metodologías tradicionalistas.

A pesar de los intentos realizados por la implementación de la lengua extranjera en diversos niveles educativos, no ha sido suficiente el avance obtenido, ya que en la formación de los futuros docentes no se ha dado prioridad a este ámbito; el programa de estudios 2012 actual de escuelas normales plantea la enseñanza de este idioma a partir del tercer semestre de licenciatura, convirtiendo a la población estudiantil en alumnos de segundo idioma, dejando a un lado los aprendizajes metodológicos que implican la enseñanza de la misma, he ahí donde radica el problema, es por ello que se plantea una propuesta de innovación en este sistema educativo, en donde se integre un aprendizaje virtual, a través de herramientas digitales en la enseñanza de la lengua extranjera, no sólo se trata de integrar las TIC en este proceso de formación, se trata de actualizar el currículum al nuevo paradigma educativo.

Los estudiantes normalistas son un sector indispensable en la sociedad, son ellos quienes se enfrentarán a la formación del nivel básico y si no se brindan las herramientas suficientes en su formación, el sistema educativo mexicano difícilmente progresará.

\subsection{El aprendizaje del idioma Inglés como lengua Extranjera}

De acuerdo a la investigación realizada por Marco Beltran (2017) el idioma Inglés ha sido considerado como el idioma de mayor uso en el mundo, como uno de los idiomas universales de comunicación, motivo por el cual diversas instituciones educativas de nuestro país lo han integrado en su currículo como materia obligatoria, posibilitando mayor oportunidades a los estudiantes en su desarrollo profesional y social. "Al hablar del Inglés como idioma extranjero se hace referencia al aprendizaje de un idioma diferente al de la lengua materna, y que además no es el que se emplea en la vida cotidiana del estudiante y el medio en el cual desarrolla sus actividades" (Mei, 2008). 
El proceso de aprendizaje del idioma Inglés como lengua extranjera se origina comúnmente en las clases formales dentro de las escuelas ya que se realizan actividades estructuradas en su formación, a pesar de que este proceso se da en su mayor parte en el ámbito educativo, los estudiantes pueden alcanzar un alto grado de desarrollo del idioma Inglés únicamente en las aulas, aunque también pueden desarrollarse con prácticas informales, como son prácticas comunicativas con personas hablantes del idioma e utilización de las TIC.

La adquisición de una lengua extranjera es un proceso de aprendizaje que sucede la mayor parte del tiempo en un ambiente de formación académica, y que se da en un lugar en el cual no se usa el idioma. Es decir, que la adquisición de una segunda lengua se da luego de que el individuo ha aprendido su lengua materna (Rojas, (s.f.)).

Entre las teorías de mayor importancia para conocer cómo se da el proceso de su adquisición de una segunda lengua se mencionarán las siguientes:

\section{METODOLOGÍA DE LA INVESTIGACIÓN}

\subsection{Población y muestra}

El estudio se realiza en las Escuelas Normales que se ubican en la región de Valles Centrales del estado de Oaxaca, las cuáles de acuerdo al plan de estudios 2012 establece la asignatura de Inglés como parte de su malla curricular: Centro Regional de Educación Normal de Oaxaca (CRENO), Escuela Normal de Educación Preescolar de Oaxaca (ENEPO) y la Escuela Normal Bilingüe Intercultural de Oaxaca (ENBIO); la encuesta se aplicó a 3 docentes que imparten la asignatura del idioma Inglés y una muestra de alumnos de diferentes licenciaturas que de acuerdo al Plan 2012 toman dicha clase. La población estudiantil muestral seleccionada fueron 50 alumnos de cuarto y sexto semestre de licenciatura, con edades comprendidas entre 19 y 23 años.

\subsection{Instrumento para recolectar información}

En este apartado se establecen los instrumentos de registro que se utilizaron para recabar información, la investigación utilizó una metodología cuantitativa y la técnica de recolección de información sobre una población a partir de una muestra, fue mediante encuestas de docentes y alumnos que de acuerdo al programa de estudios, imparten y reciben las clases de la lengua extranjera (inglés), las cuales fueron semiestructuradas, de manera presencial escrita y oral; de acuerdo con Andrés Hueso y Ma. Josep Cascant (2012) la encuesta "es mucho más que el cuestionario. Es la base sobre la que se sustenta el cuestionario. Abarca el diseño y aplicación del cuestionario (trabajo de campo) y el procesado de los datos obtenidos. Entendida como metodología con entidad propia, puede incluir también la operacionalización y el diseño muestral" (p. 28).

La encuesta se refiere a una técnica de investigación basada en las declaraciones expuestas por una muestra representativa de una población de alumnos y docentes de las Escuelas Normales de Valles Centrales, permitiendo conocer sus opiniones y actitudes de la población muestral. Considerando así la definición de Cea (1999) quien establece que la encuesta es "la aplicación o puesta en práctica de un procedimiento estandarizado para recabar información (oral o escrita) de una muestra amplia de sujetos.

La muestra ha de ser representativa de la población de interés y la información recogida se limita a la delineada por las preguntas que componen el cuestionario precodificado, diseñado al efecto". 
Las preguntas de la encuesta están divididas en tres categorías respectivamente: La primera es la Implementación de recursos digitales, la segunda categoría corresponde a la enseñanza y aprendizaje del inglés y la última es la motivación e interés por el idioma Inglés. La herramienta para alumnos normalistas se constituye de 14 preguntas y la de los docentes que imparten la asignatura de Inglés 12 preguntas, mismas que fueron video grabadas para su análisis. En la encuesta para alumnos las preguntas que se encuentran en la categoría de implementación de recursos digitales son las 2, 4, 6, 7, 9, 10 y 11; en la categoría de enseñanza y aprendizaje del Inglés se encuentran las preguntas 1, 5, 8, 9,12 y 13 , y las preguntas 3 y 14 se establecen en la categoría de motivación e interés por el idioma inglés. Con respecto a la encuesta de los docentes las preguntas 2, 3, 5, 7, 8 y 11 se encuentran en la categoría de implementación de recursos digitales, en la de enseñanza y aprendizaje del inglés se establecieron las preguntas 1, 4, 6 y 9, y en la categoría de motivación e interés por el idioma inglés se designaron la 10 y 12 .

\subsection{Procedimiento para recolectar información}

La recolección de los datos del instrumento de los estudiantes y docentes fueron contestadas de manera impresa; cada persona al leer atentamente las diversas cuestiones seleccionó la opción de respuesta que le resultara más próxima o la que mejor se ajustara a su situación, subrayando o señalando con una cruz en el recuadro correspondiente a la respuesta elegida, con la opción de poder preguntar si tuviera alguna duda. Esta aplicación se hizo de manera presencial.

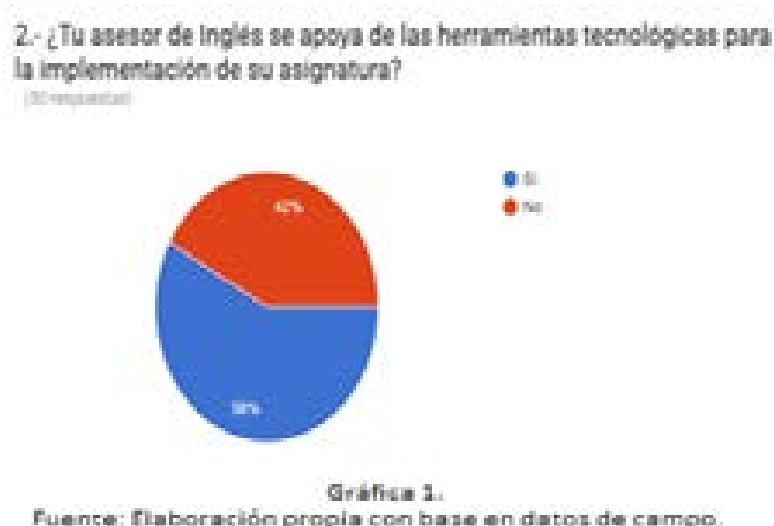

\section{DISCUSIÓN DE LOS RESULTADOS}

Una característica relevante de la encuesta es que sólo permite extraer conclusiones en términos de relaciones y no en términos causales, ya que es una estrategia de investigación descriptiva, los datos e información obtenidos en las encuestas contestadas se capturaron y procesaron en el programa Microsoft Excel, se usó la estadística descriptiva, en donde se analizaron de acuerdo a gráficas de pastel y gráficas de barras de frecuencia.

\subsection{Encuesta aplicada a alumnos}

A continuación se presentarán los resultados de la encuesta de los alumnos de acuerdo a las categorías que se establecieron:

En la categoría de implementación de recursos digitales se presentan algunos resultados de las preguntas correspondientes, aludiendo que la utilización de los recursos digitales para la enseñanza del inglés es un reto que incide en la formación del profesorado con la finalidad de valorar y usar didácticamente la tecnología, fortaleciendo el aprendizaje de los alumnos.

La gráfica 1 muestra el uso que tienen los alumnos en sus clases de Inglés de acuerdo con la implementación de los recursos digitales de los docentes, manifestando que el $58 \%$ de los docentes que les imparten o les han impartido la asignatura de Inglés en las Escuelas Normales, utilizan las herramientas tecnológicas para el 
desarrollo de sus clases, existiendo un $42 \%$ que en su metodología de enseñanza no contempla los recursos tecnológicos. La utilización de los recursos digitales en el proceso de enseñanza y aprendizaje La inclusión de estos recursos en la programación didáctica es indispensable como fuente de conocimiento y aprendizaje como medio para atender las necesidades de los estudiantes

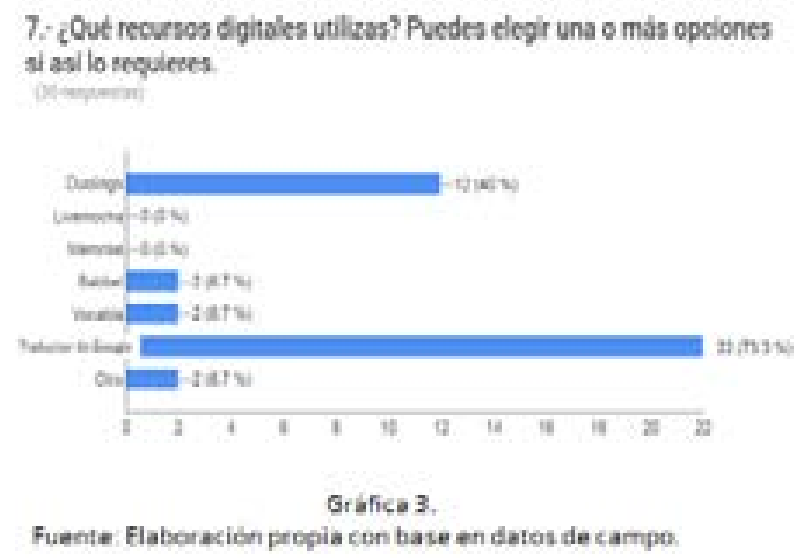

4. ¿Con que frecuencla el catedrático utiliza en wa clase de ingler. programas Sigitales que te apudan en el aprendisaje del ldioma?

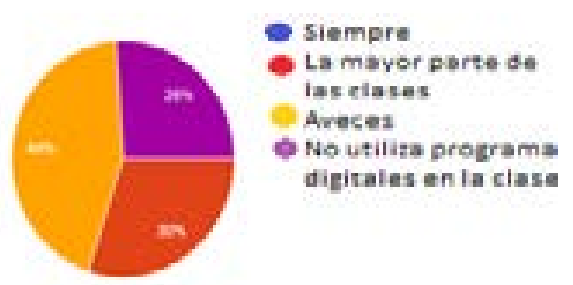

Grbica 2.

Fuenter Elaboración propia enn base en daros de campo.

En la gráfica 2 se manifiesta el $30 \%$ de la población muestral de alumnos que el docente utiliza la mayor parte de las clases programas digitales en el proceso de enseñanza y aprendizaje del Inglés, el $44 \%$ sólo a veces incorpora estos programas en sus clases y el $26 \%$ hace mención que sus docentes no utilizan programas digitales en su metodología de enseñanza.

El desarrollo de las clases de Inglés en las escuelas normales de Valles Centrales, los alumnos manifiestan la poca utilización de los recursos digitales de los docentes, demostrando así que no están inmersos en la nueva era digital, en donde la tecnología está incursionando en todos los ámbitos educativos, considero que este aspecto es importante ya que hace falta un proceso de actualización y formación, demostrando en esta gráfica el resultado de sus concepciones y competencias en la utilización de estos recursos en su planeación didáctica.

De los 30 alumnos que contestaron este cuestionamiento, en la gráfica 3 se muestra la utilización de los recursos digitales para aprender Inglés, el programa que más utilizan en un $73 \%$ es el traductor de Google, seguido de Duolingo siendo 12 personas las que hacen uso de este programa interactivo, y 2 personas hacen uso de Babbel, 2 personas de la plataforma Vocabla y el $7 \%$ de la muestra utiliza otro programa distinto para su desarrollo del aprendizaje. Las aplicaciones presentadas son recursos comercializados que le facilita el aprendizaje de una lengua extranjera, aunque también se puede apreciar que el $40 \%$ de la población muestral no contesta la pregunta haciendo mención que no hacen uso de ningún recurso digital para su formación.

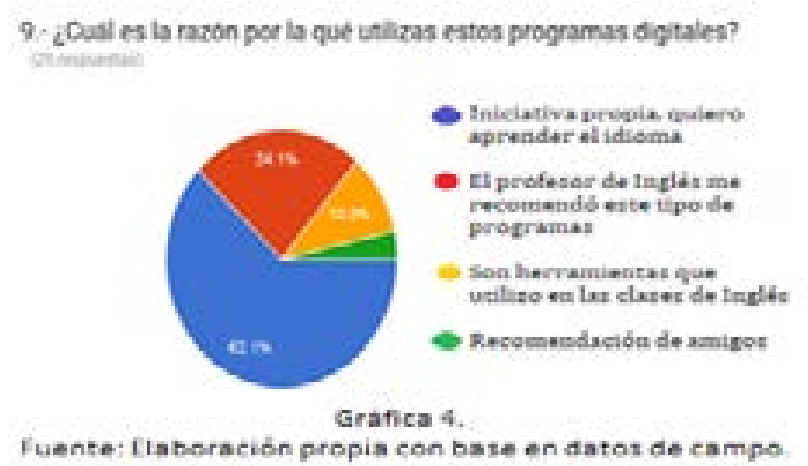

Fuente: flaboración propis con base en datos de campo. 
12. ¿Como consideras el efecto que provoca la wilizacion de los securios bigtales en el aprendicaje del ifioma ingtes? noming

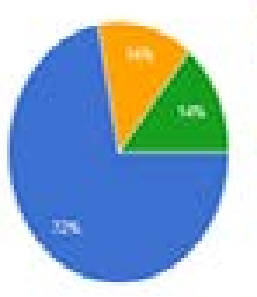

Es favorable para mi.

aprendizaje

perativamente

kprobdizaj+d+lidiems

Provoca el mismo

resultado si vetlizo o ne

los propramas digitales

en ti apreandiake

No he comprobado el

rtsultado

Gráties 5

Fuente: Elaberacion propia con base en daros de campo

Un dato interesante que se presenta en la gráfica 4 es que el $62 \%$ de alumnos que hacen uso de los recursos digitales en el aprendizaje de una lengua extranjera lo realiza por iniciativa propia, ya que está interesado en aprender Inglés, el $24 \%$ que utiliza estos programas es porque su profesor se lo recomendó, sólo el $10 \%$ utiliza estas herramientas en las clases de Inglés de acuerdo a la programación de sus docentes y solo 1 alumno implementa estos programas debido a recomendación de amigos. Sólo el $60 \%$ de los alumnos respondieron a esta pregunta, de los cuáles la mayoría de ellos está consciente de la importancia del rol del estudiante y de acuerdo con Ordorica (2010) "es uno de los principales cambios que se ha logrado en la enseñanza del idioma. Son ellos quienes desempeñan un rol más activo ya que serán los responsables del nivel de desarrollo de las habilidades del idioma y son ellos quienes deben dar una mayor iniciativa dentro del aula de clase. Para que exista la apropiación de la lengua extranjera en los alumnos es de suma importancia asumir este rol activo y autónomo favoreciendo su propio aprendizaje y desarrollo comunicativo.

En la categoría correspondiente a la enseñanza y aprendizaje del inglés se presenta la gráfica 5 manifestando el
1. ¿Te gustan las clases de Ingles?

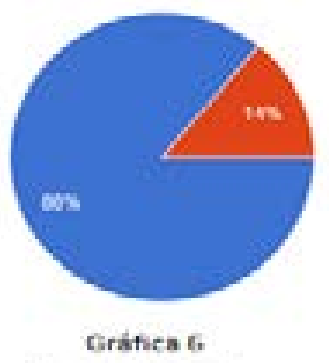

Fuente: Elaboración propia con base en datos de campo

efecto que provoca la utilización de los recursos digitales en el aprendizaje del idioma inglés desde la perspectiva de los alumnos, el cual muestra que el $72 \%$ de los normalistas que toman la clase de inglés, consideran que la utilización de los recursos digitales causa un efecto favorable para su aprendizaje, el $14 \%$ comenta que de acuerdo a su perspectiva provoca el mismo resultado si utiliza o no los programas digitales en el aprendizaje del idioma y 7 alumnos de los encuestados no ha comprobado el resultado del efecto que produce la implementación de estos recursos en su proceso de formación.

Precisando con los datos que la mayoría de los estudiantes encuestados utiliza y opina positivamente su uso, $28 \%$ restante no le da importancia respecto al uso de este tipo de recursos en el aprendizaje del idioma, mientras el estudiante tenga una participación pasiva en el proceso del aprendizaje del idioma, todo intento de desarrollo será improbable.

En la categoría correspondiente a la motivación e interés por el idioma inglés se presenta la gráfica 6 manifestando el interés que tienen los alumnos de las Escuelas Normales de aprender el idioma Inglés, en donde el mayor número de alumnos, el $86 \%$ les agrada las clases de Inglés, siendo el $14 \%$ de alumnos que no se sienten motivados por aprender esta lengua extranjera.

El Inglés como lengua extranjera, es parte de la identidad del ser humano, lo cual implica una transformación. Se trata aprender una nueva 
cultura, una nueva forma de ser, Oxford (1990) señala que "aprender un segundo idioma es a fin de cuentas aprender a ser otra persona social". Así mismo Gardner (1985) afirma que"los idiomas se diferencian de otras materias en que tienen que ver la adquisición de habilidades y patrones de comportamiento característicos de otra comunidad".

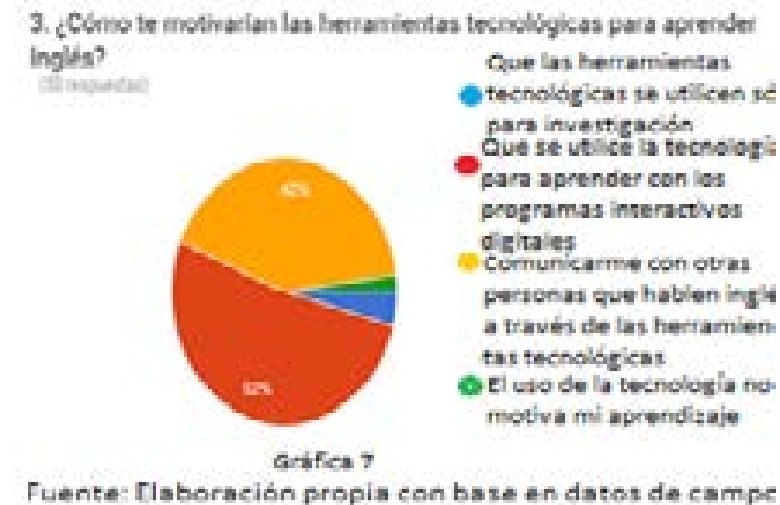

Se deduce que la gran mayoría de estudiantes comprende la importancia de aprender esta lengua extranjera, ya que en el mundo global en el que vivimos es una herramienta de trabajo, motivándolos a interesarle en desarrollar este aprendizaje, además que forma parte de su desarrollo como individuo. Aunque existe una minoría de alumnos no considera prioridad el dominio del inglés en su desarrollo profesional, esto se debe una falta de conciencia, no le atrae, el idioma no le gusta, presentando resistencia a la cultura que representa.

En la gráfica 7 muestra que la mitad de alumnos encuestados, $52 \%$, considera motivante que en sus clases de Inglés, se implementen programas interactivos virtuales en su proceso de aprendizaje, el $42 \%$ toma en cuenta el uso de la tecnología para interactuar y comunicarse con otras personas que hablen inglés, el $4 \%$ le ocasiona interés en utilizar los recursos digitales únicamente en la utilización de programas de investigación, y el $2 \%$ no le interesa utilizar la tecnología en sus clases de Inglés.

En la actualidad es indispensable que el sistema educativo se incorpore a la era digital en la cual nos encontramos, de acuerdo a Gómez, Gallardo y Macedo Buleje (2010), señalan que "las TIC son la innovación educativa del momento y permiten a los docentes y estudiantes cambios determinantes en el quehacer diario del aula y en el proceso de enseñanza aprendizaje de los mismos".

\subsection{Encuesta aplicada a docentes}

Haciendo una valoración con los docentes encuestados, los cuáles fueron 3 , únicamente, los que laboran en el CRENO, ya que por motivos externos, las demás Escuelas Normales pertenecientes a Valles Centrales, en la actualidad no cuentan con maestros especializados en impartir la asignatura de Inglés, razón por la cual fueron privados de este servicio educativo, a pesar de ser una materia indispensable de acuerdo con el Plan de estudios 2012 de las Normales.

En la categoría de la implementación de los recursos digitales en la enseñanza del idioma Inglés, los catedráticos hacen mención que utilizan recursos digitales en sus clases de lengua extranjera con la finalidad de facilitar el proceso de enseñanza y aprendizaje de los alumnos.

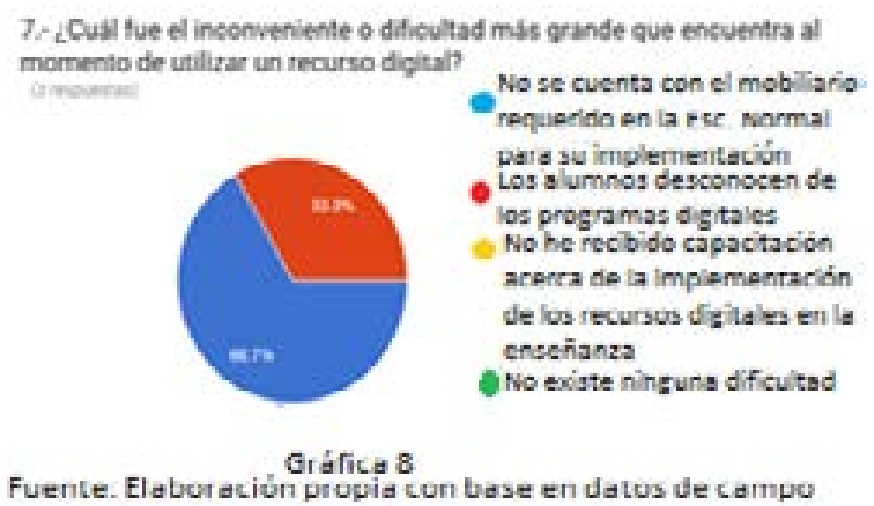

El $66 \%$ de los docentes manifiesta que han tenido dificultades para utilizar los recursos tecnológicos en su metodología de enseñanza de Inglés, siendo el $33 \%$ quien afirma que los 
alumnos no conocen los recursos digitales (gráfica 8).

Torres, Pérez y Hearn (2003) hacen mención a numerosos estudios que tratan acerca de las características y ventajas del uso de los ordenadores, desde la perspectiva del alumno:

- Aumenta en gran medida su grado de motivación

- Colabora a que el aprendizaje esté centrado en el alumno

- Favorece el aprendizaje autónomo y un papel más activo del alumno

- Promueve el aprendizaje colaborativo

- Facilita el aprendizaje de los alumnos con distintas estrategias y estilos de aprendizaje por la variedad de estímulos y respuestas multisensiorales que implica.

El uso de los ordenadores desde la perspectiva del profesor:

- Adopta un papel más de colaborador, facilitador que de instructor.

- Prescinda del aspecto más mecánico de la función evaluadora.

- Explora nuevas vías y métodos con lo que también aumenta su grado de motivación.

Una de las estrategias para enfrentar los desafíos docentes en la inclusión de los recursos digitales es la utilización de un blog de aula, materia o asignatura ya que sirve para apoyar la asignatura fuera del aula, complementar la información que da el profesorado en la misma, enviar tareas para realizar después de la actividad formativa, etc. A través de estrategias didácticas de indagación, las cuales implican programar y desarrollar acciones tendentes a que el alumnado descubra la información y elabore su propio conocimiento. Son desde una perspectiva innovadora, las que reportan resultados más fructíferos en el uso de aplicaciones de la tecnología didáctica. Este tipo de estrategias comportan, a la vez, la realización de actividades relacionadas con contenidos relativos a procedimientos, conceptos y actitudes.

Es por ello que dentro de la categoría de la enseñanza y aprendizaje del Inglés, en la gráfica 9 , todos los docentes de inglés encuestados expresan que se apoyan del programa de traductor de Google como recurso en la enseñanza del idioma, la plataforma de Duolingo es utilizado en un $66 \%$ de las clases, en cambio Babbel sólo se trabaja en un 33\%.

\section{Creve sogama digtel se apoja en la inselanzade inples? Puede degir asu omis de una opodos asila requiere.}

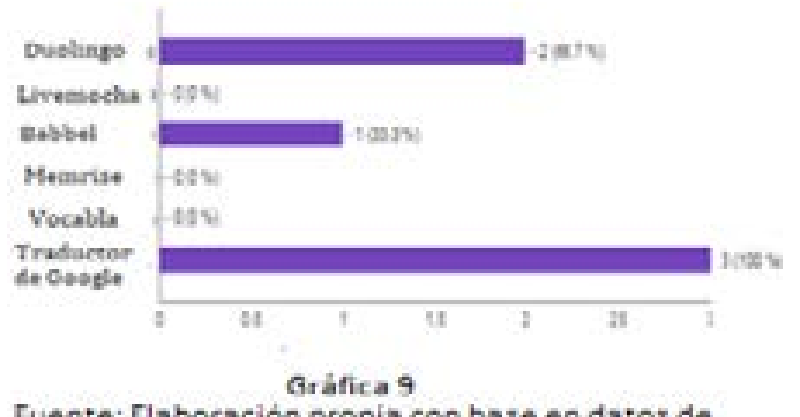

Fuente: Claboracion propia con base en datos de campo.

Las nuevas tecnologías nos permiten hacer cosas distintas a las que se hacen con las tecnologías tradicionales, pero esto no significa que las tareas se realicen de manera más rápida, automática y fiable, sino que se convierten en herramientas de apoyo para crear nuevas escenografías de aprendizaje, esto cambia el rol de profesor y el estudiante, involucrándolos en este proceso formativo.

El $66 \%$ de la población de docentes entrevistados manifiestan que a pesar de no haber asistido a cursos, investigan de manera autónoma e implementan en sus clases los recursos digitales, en comparación con 1 docente que hace mención que si asiste a 
cursos de actualización y capacitación en la implementación de los recursos digitales en la enseñanza de una lengua extranjera (Gráfica 10).

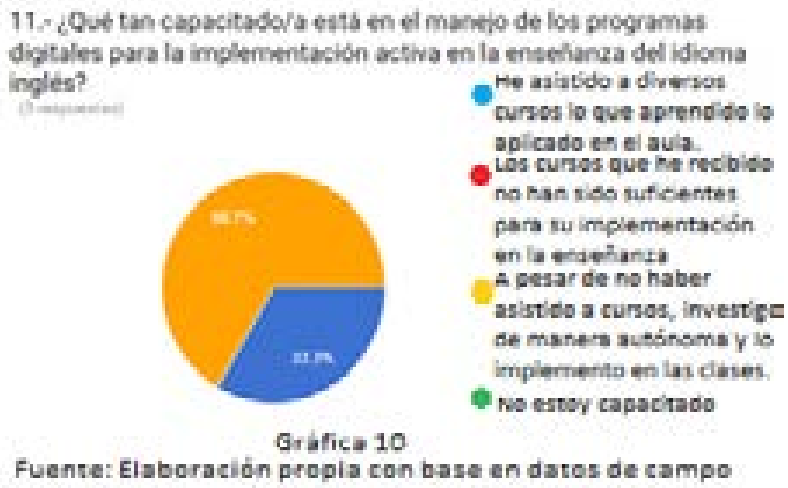

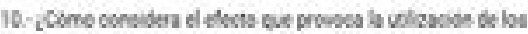

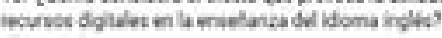

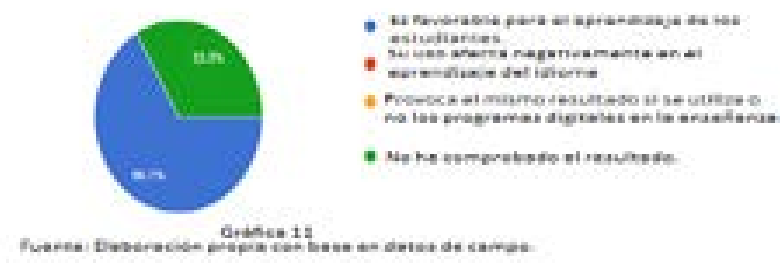

En la categoría de motivación e interés por el idioma inglés, los docentes manifiestan en la gráfica 11 que el $66 \%$ de los docentes normalistas supone que la utilización de los recursos digitales en la enseñanza del idioma Inglés produce un efecto favorable en el aprendizaje de los alumnos, mientras un docente encuestado no ha comprobado el resultado que emana su uso.

No existe un botón mágico que haga que las personas aprendan, trabajen duro $y$ actúen de una forma responsable. Sin embargo, y aunque es cierto que no exista una poción mágica para motivar a nuestros alumnos, el profesorado debe ser responsable de encontrar la manera de motivar al alumnado en su proceso de aprendizaje. Debemos trabajar sobre su motivación, incrementarla y mantenerla y para ello, debemos mejorar la calidad de nuestra práctica docente

(Ford, 1992, p. 202).

\section{CONSIDERACIONES FINALES}

La comunidad de la escuela normal se resiste a interactuar con profesionales provenientes del mismo campo o de ámbitos cercanos. Esta situación limita la posibilidad de abrirse, dialogar y enriquecer a toda la comunidad académica con base en debates y trabajo colaborativo, obstruye además procesos de investigación, el cual impide el desarrollo del sistema. Considero que uno de los problemas principales es eliminar la apatía de docentes y alumnos en incorporarse al cambio, así como permitir a docentes capacitados que impartan la asignatura de Inglés como lengua extranjera, de las tres Escuelas Normales de Valles Centrales que de acuerdo al plan de estudios 2012 establece en su currículum la asignatura de Inglés como lengua extranjera, sólo una Institución Educativa cumple con los docentes establecidos, además de que las nuevas generaciones docentes normalista no cuentan con la capacitación necesaria para la implementación de los recursos digitales como parte del proceso de enseñanza, la mayoría de los alumnos opta por tomar iniciativa propia en el aprendizaje del idioma, aprovechan los recursos que conocen e indagan para terminar su proceso de formación en este idioma.

La implementación de los recursos digitales en las prácticas formativas de la Escuela Normal recae en un modelo bancario de educación y formación en la enseñanza de inglés debido a la escasez de propuestas metodológicas que atiendan a nuevas formas de vincularse digitalmente con el conocimiento y a las actuales demandas de los entornos de enseñanza. 
Los problemas para el aprendizaje y enseñanza del dominio de este idioma se deben a causas de metodología en la enseñanza, sistematización y de práctica, el sistema educativo en cuestión de inglés no está vinculado entre secundaria y preparatoria, entonces los alumnos, a pesar de que cursan ese idioma durante seis años entre ambos niveles, no lo dominan.

Los docentes de educación básica tienen nivel de dominio mínimo o nulo del inglés, por lo que es insuficiente para cubrir la enseñanza de esa lengua desde el nivel preescolar hasta secundaria, es por ello que a pesar de implementar Programas nacionales de la enseñanza de este idioma en nivel básico, no se imparte la asignatura o es deficiente la enseñanza, debido a la escasez de educadores especializados en la enseñanza de una lengua extranjera.

Los catedráticos están convencidos que uso de las nuevas tecnologías como recursos didácticos en el proceso de enseñanza y aprendizaje facilita el aprendizaje de una lengua extranjera, pero a pesar de esta manifestación, presentan problemas metodológicos en la inclusión de estos recursos en su labor docente, considero que es indispensable y prioritario un cambio en la concepción tradicional del proceso de enseñanza y aprendizaje, además de una nueva comprensión acerca de cómo los recursos digitales pueden ayudar a crear nuevos entornos de aprendizaje en los que los alumnos se sientan más motivados $y$ comprometidos, al mismo tiempo asuman mayores responsabilidades sobre su propio aprendizaje y puedan construir con mayor independencia sus propios conocimientos de la lengua extranjera.

Las nuevas tecnologías permiten desarrollar el cambio de enseñanza en la lengua extranjera, manifestando actividades diferentes a las que se hacen con las tecnologías tradicionales, pero esto no significa que las tareas se realicen de manera más rápida, automática y fiable, sino que se convierten en herramientas de apoyo para crear ambientes favorables de aprendizaje, esto cambia el rol de profesor y el estudiante.

Los estudiantes aprenden a trabajar de dos maneras: de manera individual y en equipo. Asimismo el profesor enseña a trabajar de estas dos formas, de modo que permite ampliar los conocimientos de los estudiantes.

Es por ello que la inclusión de estos recursos en la programación didáctica es indispensable como fuente de conocimiento y aprendizaje como medio para atender las necesidades de los estudiantes y facilitar la adquisición de una lengua extranjera.

\section{REFERENCIAS BIBLIOGRÁFICAS}

Ausbel, D. A. (1963). The psychology ofmeaningfull verbal leaming: an intruduction to school. New York: Gruñe \& Stratton.

Castillejos, A. G. (2014, julio 23). Hace noventa años, en Oaxaca se fundó la primera Normal, en la época posterior a la Independencia de México. El Piñero de la Cuenca. Retrieved from http://www.elpinerodelacuenca.com.mx/epc/ index.php/oaxaca/77629--hace-90-anos-enoaxaca-se-fundo-la-primera-escuela-normalen-la-epoca-posterior-a-la-independencia-demexico.

Cea D’Ancora, M. A. (1999). Metodología cuantitativa. Estrategias y técnicas de investigación social. Madrid: Síntesis.

Chomsky, N. (1965). Aspects oftheory ofsyntax. . Cambridge: MA: I.LT. Press.

Civeria C., A. (2004). La legitimación de las escuelas normales rurales. (C. Mexiquense, Ed.) México: Colección Documentos de Investigación. 
Contreras, O. (2012). Stephen Krashen: sus aportes a la educación bilingüe. Rastros Rostros, 14, (27), 123- 124.

De Castro, A. (2009). Adquisición de Segundas Lenguas. Recuperado de https://guayacan. uninorte.edu.co/divisiones/iese/lumen/ ediciones/8/articulos/adquisicion_segundas lenguas_adecastro.pdf

Dewey, J. (1932). Mexico 1926. Progressive Education.

Dewey, J. (2011). Conferencia sobre la filosofía de la educación. Primera conferencia, (p. 2).

Fernández, M., \& Pérez, J. . (2004). Vinculación afectiva e interacción social en la infancia. Revista española de motivación y emoción, p. 31.

Gardner, R. C. (1985). Social Psychology and Language Learning:the role of Actitudes and Motivation . London: Eduard Arnold.

Gómez, G. L., \& Macedo, B., J. (2010). Hacia una mejor calidad de la gestión educativa peruana en el siglo XXI. 14(26).

González, R. F., \& Valdéz, C., H. (1994). Psicología Humanista: Actualidad y desarrollo. La Habana: Editorial de Ciencias Sociales.

IMCO. (2015). Inglés es posible. Propuesta de una Agenda Nacional . México.

Marco, B. (2017, octubre 12). El aprendizaje del idioma Inglés como lengua extranjera. Boletin redipe, Vol 6(Núm. 4). Retrieved febrero 17 , 2020, from https://revista.redipe.org/index. php/1/article/view/227

Mei, A. (2008). Cambios de paradigma en la enseñanza de Inglés como lengua extranjera: el cambio crítico y más allá. Revista Educación y Pedagogía, 2, (51), 11- 23.
Nolasco, M. (2004). Enseñanza de una Segunda Lengua. Manual Práctico para docente. España. Ideas propias.

Oxford, R. (1990). Language Learning Strategies, What Every Teacher Should Know. Heinle, Heinle: Publishers.

Podolsky, R. (2014, abril 15). La era digital y su impacto en el sector educativo. az Revista de Educaciòn y Cultura(71).

Raby, D. (1973). Los principios de la educación rural en México: El caso de Michoacán, 19151929 (Vol. XXII). México: Sobretiro de Historia Mexicana.

Ramírez. (1937). La escuela rural mexicana.

Ramírez, R. (1976). La escuela rural mexicana. México.

Richards, J., \& Rodgers, T. (2001). Approaches and Methods in Language Teaching. Cambridge University Press.

Rojas, F. \& Garduño, G. (s.f.) Adquisición de una lengua segunda desde el punto de vista la lingüística formal. Recuperado de: http:// relinguistica.azc.uam.mx/no005/06.htm

Sacristán, G. (1998). Comprender y transformar la enseñanza. Morata.

Schickedanz, J. A. (1997). Curriculum in Early Childhood. London: Allyn \& Bacon.

SEP. (2011). Documento base de la Reforma Curricular en la Educación Normal. Retrieved recuperado el 6 de julio de 2011, from http:// ed.dgespe.sep.gob.mx/consultarc/file.php/1/ documento.base.31.julio.2011.pdf

SEP. (2012). SEP Programas de estudio. Retrieved from Dirección General de Educación Superior para Profesionales de la Educación (DGESPE): http://www.dgespe.sep.gob.mx/ reforma curricular/planes/lepri/malla_curricular 
INFLUENCIA DE LOS RECURSOS DIGITALES EN LA ENSEÑANZA Y EL APRENDIZAJE DEL IDIOMA INGLÉS EN LS ESCUELAS NORMALES DE OAXACA, MÉXICO $[1$

Torres, D., Pérez, I., \& Hearn, I. (2003).

Recursos", en (eds.), . In I. Hearn, \& A. Garcés,

Didáctica del Inglés (p. 222-263). Madrid:

Pearson Prentice Hall.

UNAM. (2012). México: Dirección General de Publicaciones y Fomento Editorial, UNAM . Retrieved from http://www. planeducativonacional.unam.mx 